\title{
LES ICONES PATRIOTIQUES ET LE FORMATAGE DES ESPRITS JUVENILES (1870-1914): AXIOLOGIE DES MANUELS SCOLAIRES ET DES ALBUMS
}

\author{
Laurence OLIVIER-MESSONNIER \\ CELIS Université Blaise Pascal Clermont-Ferrand II \\ laurence.messonnier@orange.fr
}

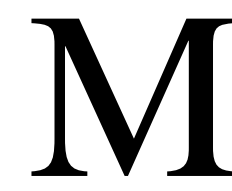

ettre en place les Instructions Officielles, en proposer une adaptation littéraire et objective pendant une période cruciale d'entre-deux-guerres comme celle de 1870-1914 tient de la gageure. L'instruction civique, l'histoire, le français et le chant s'avèrent primordiaux dans la formation des esprits juvéniles, voire dans leur formatage. Les aspirations vengeresses et l'ambition de grandeur nationale essaiment jusque dans les albums de Boutet de Monvel et de Job. Notre but est de débusquer les traces de l'exaltation cocardière, du patriotisme défensif, des témoignages d'amour national, des incitations idéologiques antigermaniques à travers les textes et les images proposés par les livres scolaires et extrascolaires, afin d'observer comment la littérature et l'iconographie prennent le relais des institutions. Magnifier les vertus comme l'abnégation, la bravoure, l'obéissance au service de sa patrie passe par la lecture d'exemples émouvants et la célébration de héros martyrs. Forger le caractère d'hommes soucieux de leurs devoirs est un gage de sécurité pour l'Etat qui n'oublie pas que l'idéal moral inculqué doit pousser ultérieurement à la reconquête des provinces annexées. Or pour favoriser le patriotisme des élèves après la défaite de 1870, l'histoire sert de figure de proue: les exemples héroïques qu'elle fournit, offrent une vision concrète du patriotisme aux enfants pour qui ce mot reste bien abstrait. Les récits édifiants des manuels d'histoire (Augé - Petit, vers 1890a et b) érigent sur un piédestal les grandes figures mythiques de Vercingétorix à Napoléon $\mathrm{I}^{\mathrm{er}}$ en passant par les soldats de l'An II; les couleurs du drapeau fédèrent textes et dessins au prix d'anachronismes révélateurs de l'entreprise idéologique que renforcent les albums de Boutet de Monvel et de Job respectivement dédiés à Jeanne d'Arc et à Napoléon I ${ }^{\text {er }}$.

\section{Révélations des manuels scolaires}

L'apprentissage du patriotisme par la lecture de récits édifiants est défendu par George Duruy dans l'ouvrage d'Emile Bocquillon (1907: XIII-LI). Il est partisan d'un enseignement de l'histoire par 
des anecdotes frappantes qui émeuvent, non par des textes documentaires sans âme. Il juge nécessaire de proposer ces livres qui «recueill[ent] pieusement, [...] propos[ent] à l'admiration de ces jeunes esprits les beaux actes de dévouement, de sacrifice, accomplis à l'ombre du drapeau» (Bocquillon, 1907: XIII). L'avantage du récit est double puisqu'il plaît et instruit à la fois. Il cumule l'attrait du divertissement et le sérieux de l'histoire militaire. L'examen de quelques manuels d'histoire que nous avons eus en notre possession révèle des nuances dans la présentation des faits, une impossible objectivité, mais plus encore, témoigne de la récurrence de figures historiques tant dans les textes que l'iconographie qui les accompagne. La conscience nationale a besoin d'être exhaussée après la défaite de 1870 pour que la France retrouve son prestige parmi les plus grandes nations.

\section{Le panthéon des guerriers libérateurs de la France}

Pour cela il faut amener dans l'esprit des élèves des images puissantes de guerriers valeureux, d'hommes politiques prestigieux et imprimer leurs actes de bravoure dans les mémoires juvéniles. Il n'est donc pas étonnant de retrouver toujours les mêmes personnages érigés en héros, notamment les guerriers. Ils sont les seuls capables de «donner aux âmes des petits Français une certaine trempe de vaillance allègre, de résolution et [...] d'orgueil» (Duruy, Bocquillon, 1907: XXI-XXXII). Là se trouve la condition sine qua non de l'accomplissement de la liberté républicaine. Tous les livres d'histoire consultés ont une forte connotation militariste quel que soit le niveau des destinataires. Le plus neutre est le Cours d'Histoire de Ducoudray (1884: 262) destiné au cours supérieur. Jeanne d'Arc y apparaît comme la sauveuse de la France, «forte du sentiment national», «l'âme visible de la patrie» (Ducoudray, 1884: 262). Au-delà de la transmission sociale du passé, les leçons d'histoire relayées par les instituteurs répertorient les héros qui ont fait la France et mettent à la portée du plus grand nombre des connaissances sous forme de propagande. Le sentiment d'appartenance à une communauté nationale est soudé au cœur par l'exposé de références qui sont autant de mythes fondateurs d'une mémoire vivante. Les vulgarisateurs de l'histoire de France ont pour nom Ernest Lavisse, Claude Augé et Maxime Petit, Gauthier et Deschamps. Historiens à vocation populaire, ils ont privilégié la diffusion et la transmission de l'Histoire par les canaux scolaires que sont les manuels. Ce faisant, ils ont instrumentalisé l'Histoire à des fins partisanes et l'ont engagée sur trois grands axes mémoriels: les combats religieux avec les croisades, les luttes politiques opposant l'Ancien Régime et la Révolution libératrice, la puissance coloniale française convergent à la source du sentiment national et exaltent de façon anachronique le patriotisme déjà présent du temps de Vercingétorix. La glorification consensuelle de la France porte sur les héros qui ont su la défendre, étendre son territoire et sa puissance dans le monde, par le biais de courts récits souvent apparentés à de brèves biographies apologétiques en forme d'hommages.

Claude Augé, neveu de Pierre Larousse, collabore avec Maxime Petit à l'écriture de plusieurs cours d'Histoire de France pour l'école primaire, dont les différents niveaux ont été réédités par Larousse de 1893 à 1923. Leur manuel d'Histoire est l'un des plus diffusés dans les écoles de la 
Troisième République après le «Petit Lavisse». Gauthier et Deschamps, Augé et Petit tiennent un discours commun sur un passé nourri de figures héroïques qui constituent ce que Christian Amalvi (2001: 50) appelle un «panthéon scolaire». L'imaginaire national de l'enfant est alimenté par des images d'Epinal peuplées de héros guerriers: Vercingétorix, Roland, Jeanne d'Arc, Bayard, Turenne, Condé, Hoche, Marceau, Gambetta. Cette galerie de personnages sera complétée par les portraits de Joffre et de Clemenceau pendant la Première Guerre Mondiale. La Révolution française est célébrée tandis que l'hymne national participe à l'élaboration de l'épopée française. Le double recours littéraire et iconographique marque mieux les esprits. Le topos du héros qui se sacrifie pour son pays illumine les récits légendaires d'une saine aura en cette période pessimiste de traumatisme d'après défaite.

La métaphore filée de la personne biologique appliquée à la France par Michelet fait florès: «La France est un soldat», a-t-il dit (Duruy, Bocquillon, 1907: XIV). Saint Louis, François Ier, Henri IV, Louis XIV, les généraux révolutionnaires, incarnent successivement cette France personnifiée. Cette divulgation est rendue possible en premier lieu par l'ouvrage d'Ernest Lavisse à l'intention de l'école

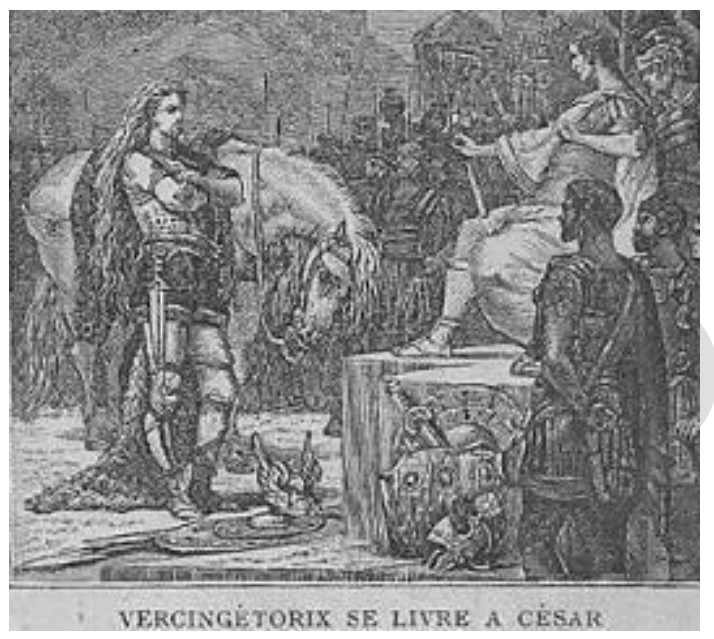

(Gauthier - Deschamps, 1905: 3) laïque où «l'histoire de France est sacralisée par les saints laïques de Vercingétorix à Gambetta» (Amalvi, 2001: 50). La prise de conscience de la nécessité d'une culture nationale est la réponse à la demande de rivalité avec l'Allemagne.

Sur le plan structurel, les manuels d'histoire destinés aux cours élémentaire et moyen utilisent volontiers la narration, et l'histoire se réduit à des récits courts vantant les exploits de héros nationaux. Contrairement à la présentation neutre du livre de cours supérieur, une ponctuation suggestive révèle la forte implication des historiens dans l'exposition. De même le fréquent recours aux questions rhétoriques prend à témoin le lecteur et appelle son adhésion: «Vercingétorix, ce "Barbare", comme l'appelait César, ne se montre-t-il pas plus grand et moins barbare que son puissant vainqueur?» (Gauthier - Deschamps, 1905:3) Les vignettes accompagnent le texte et exposent les héros dans une situation avantageuse, une pause mémorable qui insistent sur la ténacité face à l'adversaire. De même, le Premier Livre d'Histoire de France de Claude Augé et Maxime Petit propose une image de la reddition de Vercingétorix où il ne perd rien de sa superbe, droit sur son cheval (Gautier - Deschamps, 1905: 3; Augé - Petit, vers 1890a: 6). Le texte sans être aussi dithyrambique que celui du cours moyen, insiste sur la déloyauté et le manque de panache de César face à la bravoure de Vercingétorix.

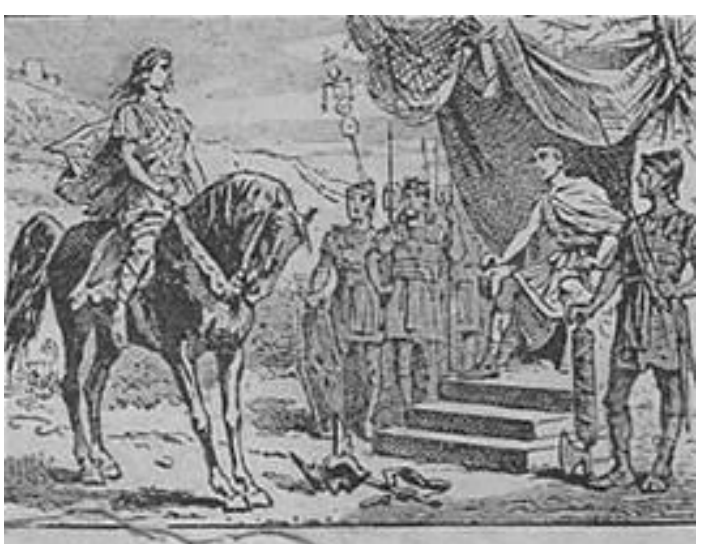

(Augé - Petit, vers 1890a: 6) 


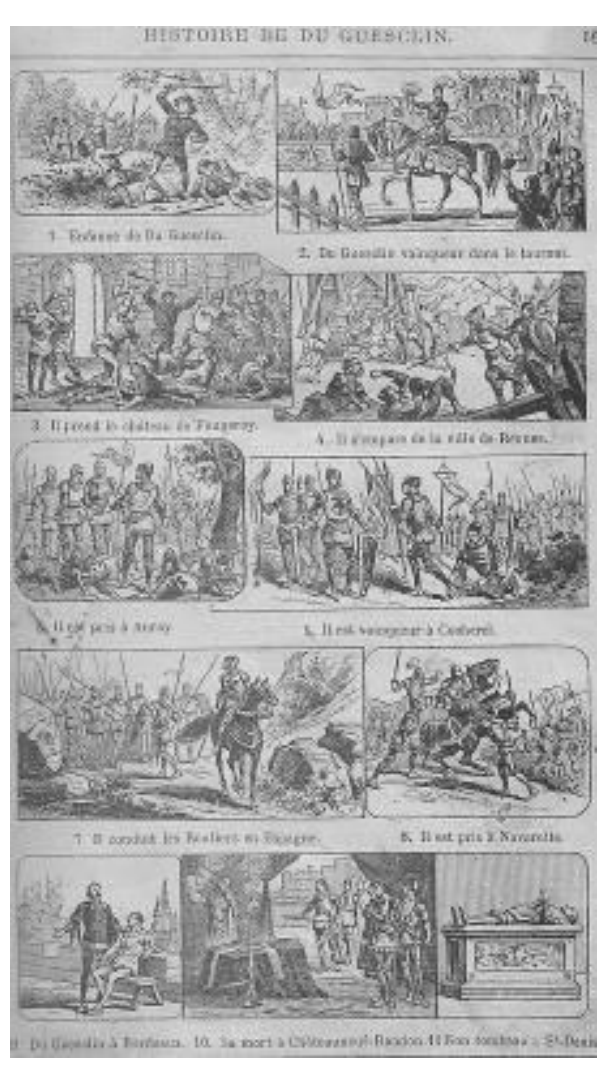

(Augé - Petit, vers 1890a: 57)

Une étude diachronique des manuels d'histoire décèle la stigmatisation des Huns et de leur chef Attila pour leur sauvagerie, thème abondamment exploité pendant la Première Guerre Mondiale: la littérature propagandiste considère la cruauté barbare comme un dangereux atavisme germanique hérité des Huns. La mort de Roland fait partie des topoï historiques propices à l'apologie des vaincus héroïques. L'épopée nationale s'enorgueillit des exploits des grands hommes et notamment des guerriers comme Roland: la défaite n'est pas envisagée comme une humiliation mais rehausse le prestige des vaincus qui ont su résister jusqu'au bout.

Devenu bréviaire national, le livre d'histoire décerne la palme à Jeanne d'Arc, Du Guesclin, Bayard en matière d'hommage rendu aux grands défenseurs de la France. L'exemplarité est accrue par la vulgarisation due aux livres de bibliothèque scolaires et aux albums comme ceux de Job ou de Boutet de Monvel. L'histoire de Du Guesclin se déroule en deux épisodes de lecture et une planche de vignettes illustratives tout comme celle de Jeanne d'Arc (Augé - Petit, vers 1890a: 57-59, 61-63).

Leur lutte sans merci contre les Anglais y est célébrée ainsi que leur ardeur au combat. La soif inextinguible de combats de Du Guesclin dès le plus jeune âge en fait un héros national. Plus remarquable est l'éloge de Jeanne d'Arc commun à tous les manuels: sa jeunesse, sa combativité ardente alliée à une pieuse douceur la sacralisent. Son supplice l'érige au rang de martyre et les images qui illustrent son épopée soulignent les scènes d'affrontement et lui dressent une stèle dans l'ultime dessin du bûcher: la sémantique patriotique s'empare des commentaires de façon anachronique en présentant l'héroïne comme celle qui a donné «un élan à la défense nationale» (Augé - Petit, vers 1890a: 64) et en ajoutant que «le mouvement patriotique dont Jeanne d'Arc a été l'âme, lui survit» (Augé - Petit, vers 1890b: 27). Jeanne d'Arc «a réveillé le sentiment du patriotisme», peut-on lire dans le manuel du cours moyen. Investie d'une mission salvatrice, sa statue a droit à une épitaphe d'Anatole France fort subjective: «Elle vécut, s'arma, mourut pour la France, et c'est ce qui nous la rend chère à tous indistinctement» (Augé - Petit, vers 1890b: 25). Le paragraphe de lecture qui succède au développement de ses exploits, est

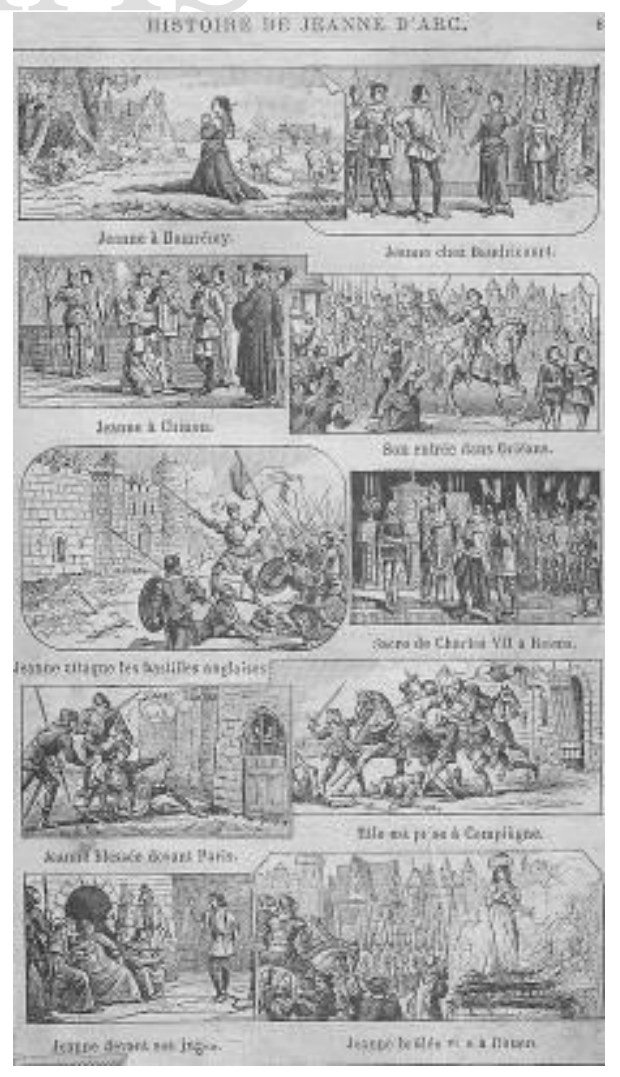

(Augé - Petit, vers 1890a: 61) 
surmonté d'un tableau en cinq médaillons, sorte de Pentateuque de la vie de la martyre. La modalité exclamative du titre, «Notre héroïne nationale!» exalte l'admiration tandis que la surenchère de superlatifs laudatifs duplique l'édification de «la plus sublime, la plus pure figure de notre Histoire nationale qui fût» (Augé - Petit, vers 1890b: 26). Les exercices de raisonnement et d'imagination qui suivent font appel au jugement des enfants dont on attend qu'ils fustigent la lâcheté et l'ingratitude de Charles VII: «Que pensez-vous de la conduite de Charles VII à son égard?», leur demande-t-on.

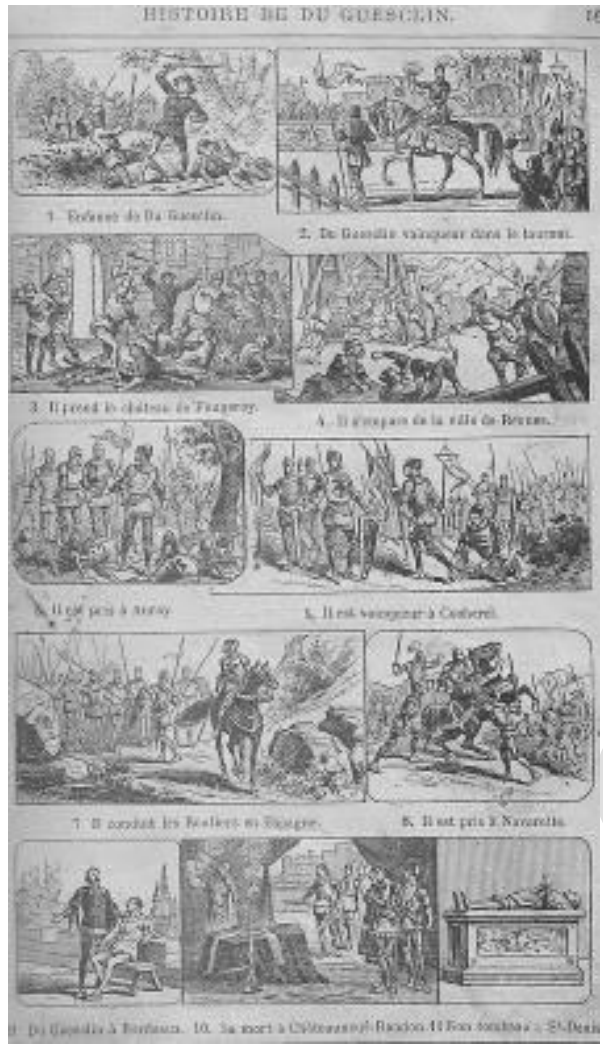

(Augé - Petit, vers 1890a: 77)

Le traumatisme de la défaite de 1870 implique de nouvelles exigences morales et patriotiques: les manuels d'histoire s'empressent de dresser un portrait dithyrambique des grandes figures françaises et de relater des récits prestigieux des épisodes marquants de notre vie nationale. Nos héros sont honorés pour leur courage certes et leur pugnacité, mais leur grandeur réside aussi dans leur magnanimité. Bayard à cet égard fait figure d'exemple: «type accompli du chevalier français et de l'honnête homme», écrivent Augé et Petit (vers 1890a: 75), «il ne se fit pas seulement admirer pour son héroïsme, ses qualités de cœur lui valurent l'estime de tous». La planche de onze vignettes (Augé - Petit, vers 1890a.: 77) ${ }^{1}$ qui illustre sa vie exemplaire ne faillit pas à la tradition iconographique: inaugurée par une image de Bayard enfant déjà habile à l'équitation, elle se clôt sur sa mort héroïque avec un geste d'ouverture du bras, de bonté à l'instant suprême. Le livre de cours élémentaire entend partir d'une explication sémantique de l'expression du «chevalier sans peur et sans reproche» tandis que celui du cours moyen propose une courte biographie élogieuse du héros, illustrée par «la mort du chevalier sans peur et sans reproche». Le sujet de rédaction est plutôt redondant, qui demande de résumer sa vie en s'inspirant de la lecture et de la gravure du manuel (Augé - Petit, vers 1890b: 41).

Les manuels d'histoire préfèrent aux philosophes des Lumières la Révolution Française dont les futurs soldats se réclament les héritiers. «La Marseillaise» donne lieu à une explication lyrique de sa genèse dans le livre de cours moyen qui met en scène «la vieille maman du poète» Rouget de L'Isle. Les héros de la France nouvelle ont pour nom Hoche, Marceau, Kléber, Carnot, Desaix (Augé - Petit, vers 1890b: 121$)^{2}$. Ils deviendront la matrice génétique des historiettes héroïques données à lire aux enfants de 1914 à 1918. Gambetta, l'âme de la Patrie, y trouve ses lettres de noblesse et rejoint le panthéon des héros par «sa brûlante activité». «Un grand souffle de patriotisme passait sur la patrie de Vercingétorix et de Jeanne d'Arc». La phrase de conclusion vaut pour une incitation au sacrifice,

\footnotetext{
${ }^{1}$ Voir l'image au sujet de Bayard.

${ }^{2}$ Une planche de treize vignettes leur est consacrée à la fin du livre de cours élémentaire, p. 121. Voir l'image.
} 
soulignée par la typographie italique: «On n'aime vraiment son pays que si l'on est prêt à lui sacrifier son repos, sa vie!» (Augé - Petit, vers 1890b: 133)

Le titre du chapitre VI emprunte à la métaphore familiale courante: «La patrie, c'est la grande famille». La genèse du sentiment patriotique est éclairée par des exemples probants comme ceux des chevaliers d'Assas et Bayard. La transposition du macrocosme national dans le microcosme familial exploite la fibre cocardière comme le fait également la fierté filiale ou parentale. L'histoire du chevalier d'Assas suffit à rappeler la lutte contre l'ennemi séculaire d'outre-Rhin en invoquant les vertus d'Assas, «sublime [...] témoignage du dévouement à la patrie». Les consignes de travail données aux élèves à l'issue de la leçon leur recommandent de donner libre cours à leurs propres sentiments de patriotisme dans une rédaction soignée. Le sentiment patriotique est inné et est fertilisé par le terreau scolaire, telle est la réponse attendue à la question posée par le chapitre VI: «Le patriotisme peut-il se former, se cultiver?» (Augé - Petit, vers 1890b: 81) Il s'entretient comme l'amour filial par la connaissance de son patrimoine et de ses ancêtres. Les référents qui doivent

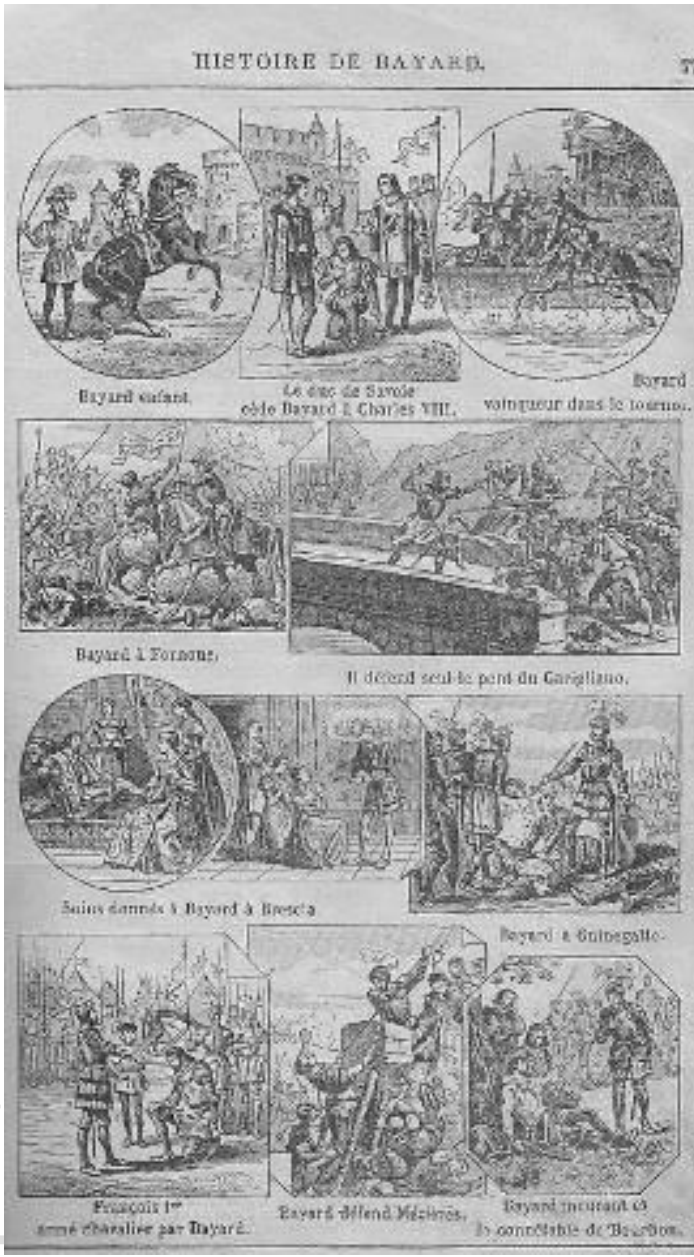

(Augé - Petit, vers 1890a: 121) faire aimer et admirer son pays, ont pour nom Charlemagne, Saint Louis, François I ${ }^{\mathrm{er}}$, Henri IV, Louis XIV, Du Guesclin, Jeanne d'Arc, Bayard, Condé, Turenne, Hoche, Marceau, Bonaparte. La connaissance de l'histoire nationale comme celle de l'histoire locale est le creuset du patriotisme. C'est ce que suggère la consigne donnée au maître par les indices d'énonciation héroïques: «Dire comment toutes deux contribuent à former le patriotisme des enfants, en leur faisant connaître les belles actions des héros et des bienfaiteurs de la France» (Augé - Petit, vers 1890b: 85).

\section{Les exigences morales d'un patriotisme humaniste}

Force est de reconnaître que le livre d'instruction civique d'E. Primaire (1902) prêche un idéal de tolérance fondé sur la devise républicaine et illustré par la poésie hugolienne: «Peuple, aimez. On devient lumineux en aimant». (Primaire, 1902: 137) ${ }^{3}$ L'idéal guerrier tombe aux oubliettes et la guerre est pointée du doigt: «La guerre est une monstrueuse iniquité, parce qu'elle est le triomphe de la force non du droit» (Primaire, 1902: 175). L'auteur différencie les guerres légitimes, le sacrifice de la vie à la patrie, des guerres de conquêtes menant aux massacres et à l'extermination. Les lauriers sont

\footnotetext{
${ }^{3}$ «Le Pape» de Victor Hugo.
} 
décernés à l'héroïque Jeanne d'Arc, aux volontaires de 1792 tandis que Charles VII, Louis XII, François $\mathrm{I}^{\mathrm{er}}$, Louis XIV, Napoléon $\mathrm{I}^{\mathrm{er}}$ sont voués aux gémonies pour leurs folles entreprises. Le droit des peuples est sacré et prime n'importe quelle guerre.

Les deux manuels de chant dont nous disposions confirment l'ardent républicanisme dont ils se font l'écho. Le chant choral de Jules Combarieu (1914) et Chants populaires pour les Ecoles de Maurice Bouchor (1913) offrent un vaste panorama républicain, allant du simple patriotisme au fervent nationalisme en passant par l'exaltation cocardière issue de la Révolution. On peut distinguer les pièces qui font la part belle à l'histoire nationale et le ferment libérateur qu'elle a vu germer. Béranger les met en valeur dans «Les Gaulois et les Francs» (Combarieu, 1914: 48), «La Fayette en Amérique» (Combarieu, 1914: 46), Palestrina dans «Révolution» (Combarieu, 1914: 16). Les chants exhaussent le prestige de la France à travers la prestance de ses héros comme La Fayette, libérateur de l'Amérique, homme des deux mondes. Les paroles résonnent comme un appel à la liberté: «Qu'a-t-il donc fait? Il a brisé les fers!» (Combarieu, 1914: 46) Les vieux démons revanchards agitent quelques couplets qui rappellent l'échec d'Attila face aux Gaulois, les envies suscitées par la France, les convoitises de nos voisins russes ou saxons, l'indignation face aux Prussiens.

Chants populaires pour les Ecoles use d'un procédé plus familier et plus grossier pour apprendre la musique aux écoliers: Sur trente-sept chants, onze entonnent l'antienne patriotique. Environ un tiers du manuel met les écoliers au pas sur l'air d'une marche à l'instar du «Chant des écoliers français» qui prépare les élèves à devenir de vaillants soldats: «La France aura besoin de nous bientôt», clament en chœur les enfants. Le dernier couplet offre un excellent exemple d'embrigadement des esprits par le lyrisme: le rythme syncopé, les allitérations rauques favorisent l'harmonie imitative en suggérant le roulement du tambour et le défilé des écoliers sur l'air de la «Marche de Turenne» (Bouchor, 1913: 34):

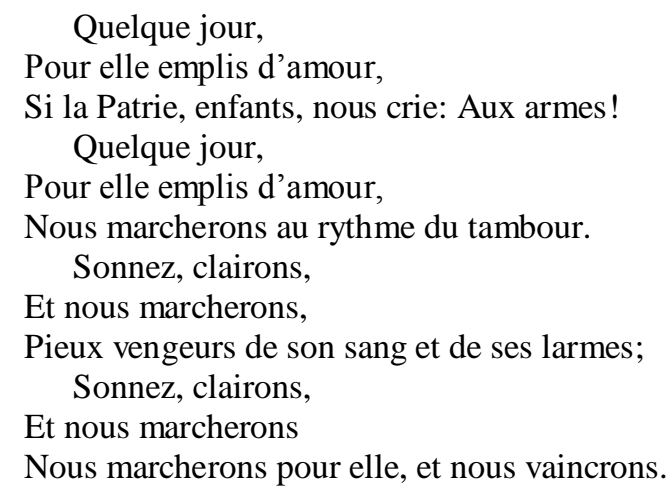

Tous les textes patriotiques rencontrés dans cet opuscule de quarante pages sont à l'image de ce poème chanté, vindicatifs et guerriers. Ils convoquent sur l'autel de la mémoire, les mânes de Jeanne d'Arc, de Bayard, de Turenne et incitent les enfants à en être les dignes héritiers. Les impératifs pleuvent, les métaphores épiques abondent, le soldat français rayonne au firmament des images d'Epinal avec sa gourde et sa pipe. Les consignes pédagogiques insistent sur le fait qu'il faut chanter avec «beaucoup d'énergie» les trois derniers vers du «Soldat français» ( Bouchor, 1913: 8): 
Gloire au drapeau.

J'aimerais bien revoir la France,

Mais bravement mourir est beau.

Les textes de lecture et de dictée concoctés par Claude Augé font écho aux leçons d'histoire et s'attardent sur la légende de la mort de Roland (Augé, 1901: 16), la loyauté de Du Guesclin (Augé, 1901: 87), de Bayard (Augé, 1901: 133), de Dumouriez (Augé, 1901: 159), de Kléber (Augé, 1901: 172), du petit caporal Bonaparte (Augé, 1901: 186), de Jeanne d'Arc (Augé, 1901: 169), héroïne phare,

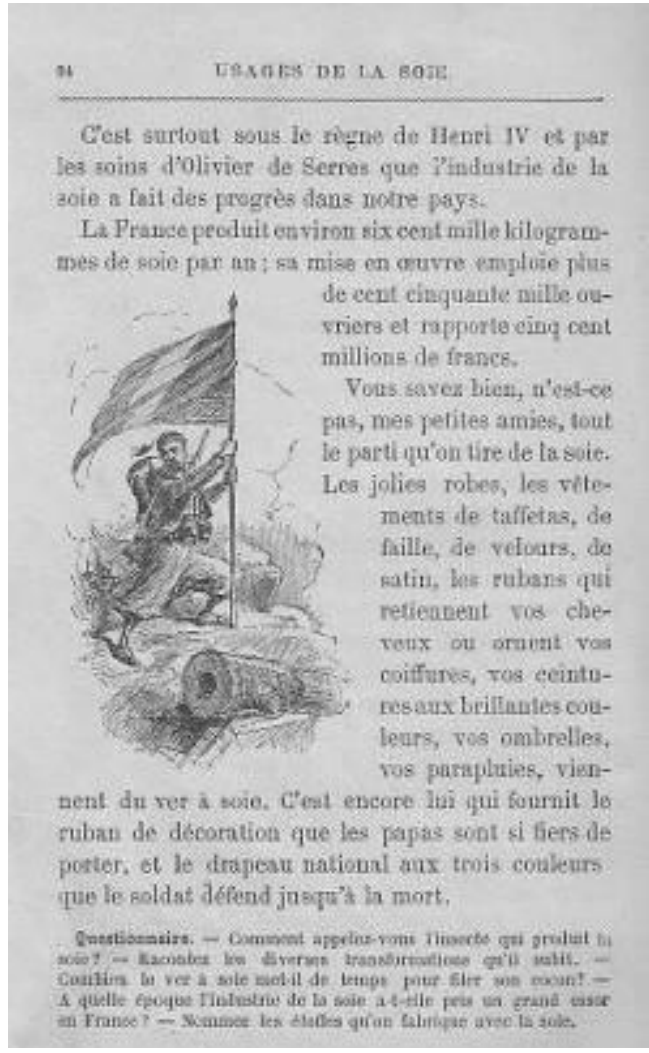

(Juranville, s. d.: 94) icône patriotique avant l'heure. Les serviteurs de la Révolution sont l'objet de tout un texte sur les pluriels exaltant les vertus des Kellermann, des Jourdan. La clausule emphatique correspond à un cri de victoire et dresse un arc de triomphe à tous les libérateurs français car «conduits par de tels hommes, nos soldats triomphèrent sous tous les ciels de l'Europe, et c'est le monde entier qui bénéficie des principes généreux de la Révolution française» (Augé, 1901: 197).

La morale patriotique scande régulièrement les exercices de lecture et les dictées. Le procédé le plus utilisé est le recours au texte informatif qui définit le terme abstrait de «patrie» par des métaphores naturelles ou humaines renforcées par les allusions au drapeau fédérateur: «La patrie, [...] c'est l'unité de notre magnifique territoire [...], c'est la gloire ineffaçable de nos pères [...], c'est la grandeur de la liberté. C'est l'ensemble indivisible de nos concitoyens. C'est la nation $[\ldots]$ que vous devez honorer [...] de votre intelligence» (Augé, 1901: 36).

\section{Le texte et le dessin aux couleurs du drapeau}

La méthode des thèmes d'étude est florissante et fort judicieuse pour mettre en exergue les richesses de la France comme les filatures de Rouen par exemple. L'évocation de l'industrie de la soie est l'occasion d'exhiber l'étendard tricolore, puisque le ver à soie «fournit le ruban de décoration que les papas sont si fiers de porter, et le drapeau national aux trois couleurs que le soldat défend jusqu'à la mort» (Juranville, s. d.: 94). La pertinence de l'illustration explicite les périphrases laudatives du texte par le dessin d'un zouave brandissant l'oriflamme à côté d'un canon. Une leçon sur les métaux et l'acier rappelle la

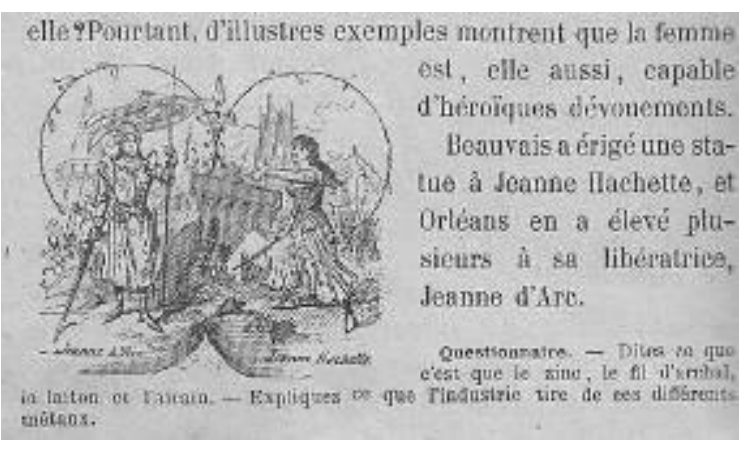

(Juranville, s. d.: 192) 
fabrication des canons avec vignette à l'appui en regard. L'airain associé aux statues met sur un même piédestal Pierre Larousse, Jeanne Hachette (Juranville, s. d.: 192) ( $^{4}$ Jeanne d'Arc deux sauveuses coulées dans le même bronze que l'homme de lettres, et fixées dans une pause altière et hiératique.

\section{Toutey et Michelet dessinent les socles de la pensée patriotique}

Le livre de l'Inspecteur primaire Emile Toutey est composé de «deux cents morceaux choisis d'auteurs français avec des explications, des questions et des devoirs» (Toutey, 1907; voir l'image de la page de garde). L'observation globale du manuel met en relief le désir constant d'accorder des textes de haute tenue littéraire à une progression annuelle qui étudie successivement chaque trimestre, la morale, l'histoire, les sciences et la géographie selon une complexité croissante. L'adaptation aux saisons reste un critère de choix pour les textes ${ }^{5}$ destinés aux enfants de onze à douze ans. La formation générale de l'esprit figure au premier rang des préoccupations et c'est dans cette optique que les exercices complémentaires de chaque lecture offrent des explications sobres et précises, des questions et des analyses d'idées pour «bien pénétrer le texte» (Toutey, 1907: IV) et surtout appréhender l'art de l'écriture selon les

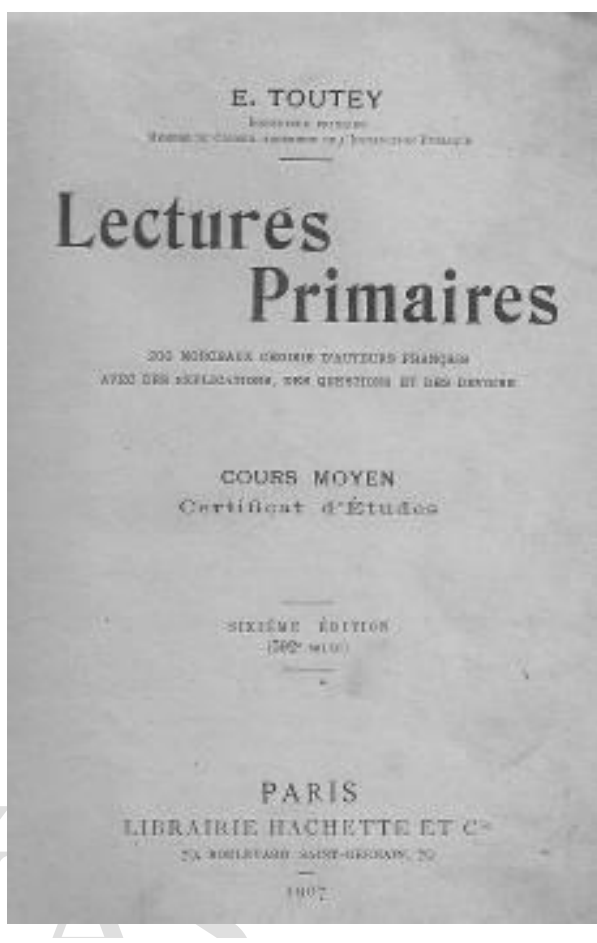

(Toutey, 1907: page de garde) meilleurs modèles littéraires. Des devoirs d'élocution et de rédaction vérifient la compréhension et visent à développer l'intelligence et l'imagination des lecteurs par des compositions proches de la réécriture. L'approche axiologique du livre est guidée par les titres ajoutés en guise de chapeau aux

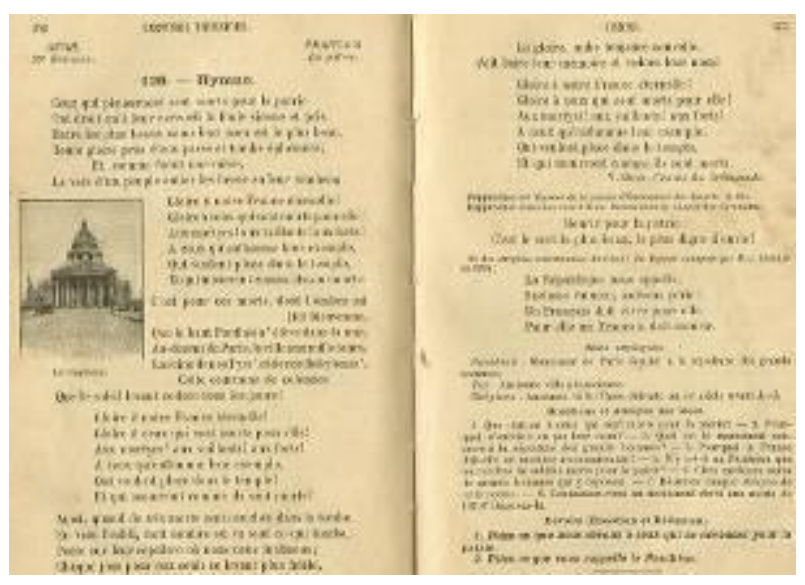

(Toutey, 1907: 252) extraits choisis: les valeurs morales liées à la famille, les critères de sélection d'un bon livre et l'incitation à la lecture, les hagiographies des grands hommes, l'apologie de la République née de la Révolution conduisent à l’ «Hymne» à la France de Victor Hugo, tiré des Chants du Crépuscule (Toutey, 1907: 252) ${ }^{6}$.

Quels que soient les auteurs célèbres mentionnés, le choix des textes historiques trahit une partialité cocardière. Certes Vercingétorix, Turenne, les vainqueurs de la Bastille, les soldats de la République, de la Révolution, de l'An II, les

\footnotetext{
${ }^{4}$ Voir les images. Jeanne Hachette est une héroïne française qui défendit Beauvais, assiégée par Charles le Téméraire en 1472. Elle tient son surnom du moyen de défense qu'elle utilisa.

${ }^{5}$ Vingt textes par mois, cinq par semaine, un par jour.

${ }^{6}$ Poème de Victor Hugo.
} 
volontaires de 92, occupent une place de choix comme dans les livres d'histoire. Cependant les auteurs des textes à l'instar de Duruy, Michelet, Thiers, Quinet révèlent un parti pris d'exaltation patriotique de la part de Toutey.

Littéralement canonisé par la Troisième République, Michelet l'historien, est encensé pour sa collaboration à l'instauration d'idées libérales et démocratiques avec son ami Quinet. Son amour pour la France qu'il personnifie en fait une entité qui progresse par elle-même, qui se recrée indéfectiblement. Sa sensibilité et son extrême bonté habitent des textes émouvants sur Jeanne d'Arc, la prise de la Bastille et la Révolution. Son esprit anime tout le livre de Toutey qui croit en l'unité fondamentale de l'humanité. Un élan vital souffle dans ses textes sur la conception de la nation. Les textes de morale sur le thème du «dévouement à la patrie» accordent une large part à la Révolution et aux soldats de la République comme le prouve le poème d'Emmanuel des Essarts «Aux soldats de la République» (Toutey, 1907: 234-235), véritable hymne à la gloire des armées de la République où coule à flots le courant épique des lauriers décernés aux «humbles défenseurs de notre France» (Toutey, 1907: 234)

Le parti pris patriotique est trahi par l'encensoir des chapeaux précédant les textes sur les Volontaires de 92: «Tous les écrivains ont célébré cet admirable mouvement des Français se levant contre l'Europe menaçante» (Toutey, 1907: 242). Le lyrisme des «Soldats de l'An II» de Victor Hugo a pour pendant le souffle épique des «Volontaires de 92» de Michelet.

\section{Des soldats de l'An II aux Poilus de 1914: la célébration de la liberté}

Le bilan axiologique que l'on peut tirer de ces analyses de manuels scolaires utilisés entre 1870 et 1914 explique en grande partie le stoïcisme des soldats de 1914, avatars modernes des soldats de l'An II. Les hommes capables de lutter pied à pied dans des conditions épouvantables pour défendre leur pays ont été formés par les «hussards noirs de la République» mais surtout par la littérature historique qui leur a été donnée à lire, à comprendre et à assimiler. Ces hommes qui n'étaient pas de vrais militaires mais de vrais combattants se sont transformés en courageux guerriers grâce à un enseignement qui les a insidieusement orientés vers les préceptes de défense et de revanche. L'humiliation de 1871 devait être lavée. Seule la morale de la raison et du devoir transmise par Hugo et les historiens de la seconde moitié du $19^{\mathrm{e}}$ siècle, a pu forger des esprits soumis à l'autorité. La contestation étant impensable, les pédagogues ont emboîté le pas aux hommes politiques. De fait la majorité des auteurs de manuels scolaires a œuvré à la préparation mentale belliqueuse des enfants. Les textes lus, de l'abécédaire au poème lyrique en passant par le document historique sont censés conjurer les faiblesses humaines inhérentes à toute société.

L'objectif n'était pas aussi chimérique qu'il paraissait au premier abord puisque force est de reconnaître que l'école et les manuels scolaires ont constitué la base d'un édifice patriotique. Toutefois il serait faux de prétendre que l'école et les manuels sont responsables de la guerre. Ils ont œuvré à la formation des esprits patriotiques par l'exacerbation cocardière et parfois à l'animosité 
antigermanique. L'orientation idéologique orchestrée par l'application des principes de la politique scolaire établis par les programmes de 1882, a persuadé les élèves que le premier devoir d'un homme est de servir sa patrie au prix de son sang. Rarement amenés à discuter, à comparer ou à choisir, les écoliers ont pris l'habitude de disserter, soutenir une argumentation déjà développée. Le métier de l'enseignement a encensé pendant quarante-quatre ans le métier des armes à travers une littérature diversifiée mais dont 1'optimisme a été jugé excessif par Jaurès ${ }^{7}$.

Le patriotisme exacerbé des dix années qui ont suivi la défaite de 1870 a mué en un idéal plus généreux et pacifiste. L'épuration du sentiment cocardier mène progressivement à la nécessité de servir la cause de la France. La place accordée aux vertus guerrières marque le pas et cède la place aux héros français bienfaiteurs de l'humanité comme Bernard Palissy, Louis Pasteur, Victor Hugo. Nonobstant cette vision pacifiste, les ouvrages dont nous disposions concernant la première décennie du $20^{\mathrm{e}}$ siècle ne démentent pas l'utilité de l'armée. Les bataillons scolaires ont disparu mais les textes exaltant l'enrégimentement des enfants persistent. Cependant les manifestations patriotiques prennent d'autres apparences: nombre de manuels célèbrent le service civil de la patrie et le civisme de l'écolier studieux. La dureté du métier de soldat est reconnue sans nuire à son panache. Les livres scolaires séparent le bon grain de l'ivraie en distinguant le patriotisme qui réjouit le cœur par son humanisme, du patriotisme guerrier et violent qui engendre des massacres. C'est pourquoi Louis XIV et Napoléon I ${ }^{\text {er }}$ sont stigmatisés dans certains manuels pour leur ambition guerrière offensive alors que les luttes de Jeanne d'Arc contre les Anglais, de Kellermann à Valmy, de Gambetta sont soumises à l'admiration des élèves.

Les poèmes, les récits et les documents historiques incitent à la militarisation de l'enfant pour qu'il défende le sol français. Toutefois, jamais un appel direct à la guerre contre l'Allemagne n'a été rencontré, seule une promesse de retour des provinces perdues à la mère patrie demeure. Les anecdotes priment et les livres préfèrent éluder les extrêmes de la guerre et du renoncement. Seule une guerre défensive peut être envisagée, la France étant trop honnête et réfléchie pour en être l'instigatrice. Cette théorie de la responsabilité criminelle de l'Allemagne dans le déclenchement de la Première Guerre Mondiale alimente toute la lecture enfantine de guerre que nous avons consultée pour la période de 1914 à 1918. La France érigée en parangon du droit et de la justice ne saurait engager les hostilités.

Là se situe la stricte corrélation entre les productions enfantines écrites et la voix officielle, qui retentit lors des obsèques de Jaurès lorsque Léon Jouhaux déclare le 4 août 1914 que «nous sommes

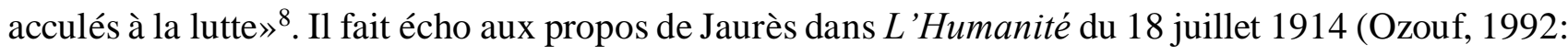
212):

Quoiqu'en disent nos adversaires, il n' y a aucune contradiction à faire l'effort maximum pour assurer la paix, et si cette guerre éclate malgré nous, à faire l'effort maximum pour assurer, dans l'horrible tourmente, l'indépendance et l'intégrité de la nation. ${ }^{9}$

\footnotetext{
${ }^{7}$ Jean Jaurès, discours de janvier 1910 à la Chambre des Députés, intitulé «Pour la laïque».

${ }^{8}$ Discours de Léon Jouhaux aux obsèques de Jaurès.

${ }^{9}$ Jaurès cité par Mona Ozouf dans La république des instituteurs.
} 
La littérature enfantine scolaire et extrascolaire se trouve propulsée au centre d'une lutte d'intérêts éditoriaux et idéologiques. Elle devient un enjeu politique au fil des quarante-quatre années séparant les deux guerres.

Selon Mona Ozouf, l'école n'est certainement pas le lieu où peut se préparer la revanche (Ozouf, 1984). Nous nuançons cette opinion car la littérature scolaire et parascolaire a su forger, via l'interdisciplinarité, un esprit de résistance et d'abnégation chez des élèves à qui on a inculqué le devoir d'obéissance et d'attachement aux racines. Les ouvrages extrascolaires ne demeurent pas en reste et exhalent des sentiments cocardiers de façon plus ostensible: y résonnent des échos militaristes, bellicistes et cocardiers dont l'incidence sur la mentalité juvénile est indiscutable. Négliger l'impact de l'école sur les futurs Poilus revient à nier la valeur axiologique des ouvrages qui leur furent soumis entre 1870 et 1914, à éluder la portée sociétale et idéologique des textes lus et appris. Sans la littérature de jeunesse, la translation de la voix officielle à la voix enfantine n'aurait jamais eu lieu. Les albums de jeunesse que nous avons lus ont généré une rémanence patriotique.

\section{Albums à thèmes: une propédeutique à l'art martial}

Des illustrateurs comme Boutet de Monvel et Job sont conscients du pouvoir éducatif de l'image. Ils rendent hommage aux grandes figures héroïques de l'histoire de France honorées par les manuels d'histoire. Faut-il y voir un signe de leur adhésion à la mentalité patriotique diffusée par l'Etat? Leur personnalité, la genèse de leurs ouvrages et leur langage iconographique divulguent les penchants nationalistes des auteurs.

\section{Boutet de Monvel: Jeanne d'Arc, icône patriotique}

Boutet de Monvel $(1896)^{10}$ doit sa célébrité aux livres illustrés qu'il crée pour le public enfantin. Dans le cadre de nos recherches sur l'imprégnation cocardière, nous nous sommes intéressés à Jeanne d'Arc, paru en 1896. La consultation de ce beau livre à couverture beige fleurdelisée nous a dévoilé des trésors d'ingéniosité et de virtuosité littéraires et iconographiques. Le titre en lettres d'or surmontées de couronnes royales et d'une épée érige la sauveuse de la France au rang d'une reine, équivalent de son édification en icône patriotique par les manuels

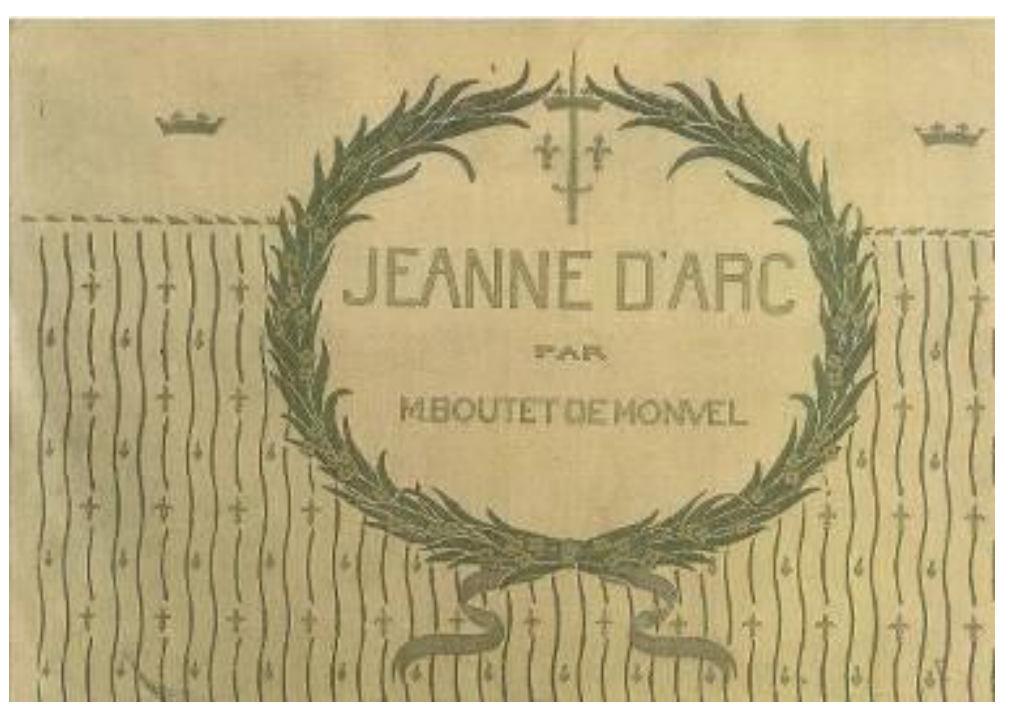

(Boutet de Monvel, 1896: page de couverture)

\footnotetext{
${ }^{10}$ Boutet de Monvel (1884-1949) est un peintre aquafortiste et un dessinateur français.
} 
scolaires. Boutet de Monvel explique d'ailleurs dans l'avant-propos daté d'avril 1896, que la couronne rappelle la gloire et l'hommage rendus à Jeanne d'Arc, un des piliers de l'histoire de France racontée aux enfants. Elle fait partie des héros séculaires «panthéonisés» par l'institution scolaire.

Pour cela, l'auteur illustrateur n'hésite pas à jouer sur les anachronismes afin d'ancrer la figure salvatrice dans les mémoires ju véniles. Dès la première page, les siècles se confondent puisque Jeanne d'Arc est à la tête d'une troupe de fantassins. La fonction allégorique de l'image est soutenue par l'exactitude des costumes et l'opposition signifiée par l'armure médiévale de l'hérö̈ne guidant la troupe en pantalons garance, redingote bleue et casquette rouge et bleue. L'anachronisme est dépassé par le message de force et d'union lancé par cette gravure: l'écrasement de l'Allemagne est signalé par la vision de Jeanne d'Arc, visière du heaume relevée, yeux tournés vers le ciel, foulant au pied les soldats ennemis. L'Allemand remplace l'Anglais. Le Prussien mis à mal a déjà le visage de la mort. La majesté du cheval de la sauveuse est accentuée par sa démarche solennelle. Le symbole est fort: l'armée française se rallie à Jeanne d'Arc, emblème de courage et de vaillance, qui lutte au nom de Dieu pour sauver la France. La puissance sémiotique vient d'une double inspiration patriotique et contemporaine pour la partie gauche et extrême droite du tableau, historique pour la partie centrale. Le dessin s'appuie sur la métonymie et l'allégorie de la France libérée. Le clairon qui sort de terre représente le soldat allemand mort dans l'enfouissement de la violence armée tandis que l'uniforme des fantassins reflète la patrie française.

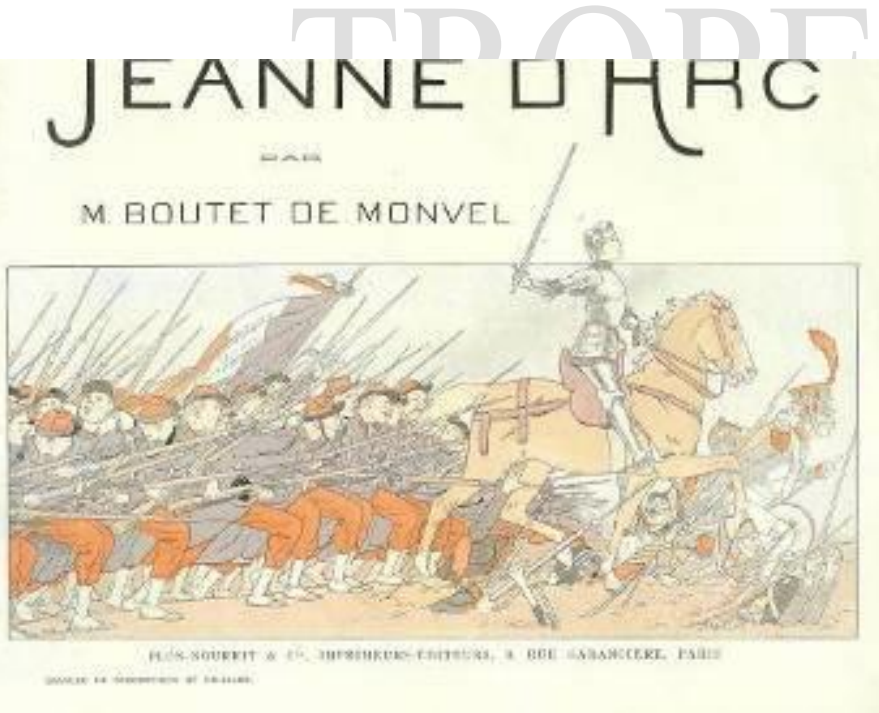

Le livre se présente comme une biographie élogieuse de la petite bergère auréolée, dans une robe aux fleurs d'oranger. Boutet de Monvel réussit le consensus religieux et républicain dans une captatio adressée aux enfants qui rend hommage à la «Sainte de la patrie», à la «martyre». Par l'alliance de la religion et de l'histoire, l'acte de lecture devient acte de patriotisme. Le mimétisme enjoint d'avoir foi en la victoire à l'instar de Jeanne d'Arc. Le conteur convainc d'autant mieux les lecteurs qu'il les installe dans une relation de confiance par l'apostrophe «mes chers enfants», devenant leur instituteur et leur proche. Il rejoint la cohorte des écrivains, substituts familiaux qui tissent une relation d'influence avec les enfants. Le destinataire doit se sentir proche de Jeanne d'Arc qui avait treize ans lorsque lui apparut l'archange Saint Michel.

L'énonciation exhorte à l'imitation, et participe de l'entreprise de conditionnement des esprits au même titre que les grands thèmes récurrents de la littérature patriotique. La gradation hyperbolique de la «patronne de la France» en «sainte de la patrie comme elle en a été la martyre», unit l'Eglise et la République, la dévotion et le patriotisme sous l'étendard tricolore. Psychologiquement, l'enfant est 
alerté par la sublimation de l'héroïne par la souffrance. La douleur transcende, et sa verbalisation exorcise les craintes pour n'en dégager que l'admiration. Le futur prometteur et convaincant employé par l'auteur attribue à son message un caractère prophétique: «L'histoire vous dira», promet-il. L'enfant est sollicité avant l'heure, promis à un avenir militaire, fondé sur la solidarité et le sentiment national. «Le jour où le pays aura besoin de votre courage» fait peser sur les épaules du jeune lecteur la responsabilité de l'avenir. Les apostrophes et les tournures à valeur impérative -«souvenez-vousen», «il faut avoir la foi»- constituent l'album en leçon de civisme et de patriotisme.

Les enfants appelés à devenir les futurs défenseurs de la France sont placés en position de contemplateurs face à la jeune paysanne terrassant le lion anglais. L'épée et la quenouille qui l'entourent (Boutet de Monvel, 1896: 3) renforcent la valeur allégorique de l'image, de la guerre et de la paix. Le résumé historique des causes de la Guerre de Cent Ans trahit la partialité du conteur dans l'emploi des termes péjoratifs à l'encontre du dauphin Charles VII. Les anachronismes lexicaux exhaussent le prestige de l'héroïne qui voulut éviter à «notre race [...] [de] perdre sa nationalité» et révèle l'orgueil cocardier de Boutet de Monvel. Il s'appuie sur les contrastes dus à la féminité et à la force de caractère de l'héroïne, il offre aux enfants un moyen de disculpation face aux faiblesses inhérentes à leur âge. A l'instar de Jeanne d'Arc, ils peuvent faire de leur «frêle patrie» un «rempart face à tant de détresses». Le parallèle historique s'accompagne d'une translation psychologique. La pusillanimité est rejetée et c'est dans l'innocence et la pureté qu'il faut puiser le dynamisme, la foi en la victoire. Le trait récurrent qui parcourt l'œuvre de Boutet de Monvel appartient au topos de la «grandeur du petit». Défi aux esprits vétilleux et mesquins vis-à-vis des plus faibles, femmes et enfants, Jeanne d'Arc n'est pas seulement l'allégorie de la France qui résiste, mais aussi l'incarnation de la force du faible. La révélation aux jeunes Français de l'existence du courage qui stagne au fond de leur cœur précède une épiphanie de vengeance. Le corollaire de la défaite de 1870 est la foi en une revanche victorieuse.

Pour rendre l'hagiographie de Jeanne d'Arc attrayante, Boutet de Monvel allie l'exigence de l'historien à l'ingéniosité du conteur. Le schéma quinaire du récit reprend les étapes qui jalonnent l'existence de Jeanne d'Arc et transforme sa vie en épopée ${ }^{11}$. La dichotomie manichéenne repose sur la grandeur de l'humble Jeanne d'Arc et la lâcheté des grands. Le genre du récit ressortit à l'épopée dont la voix fait résonner les échos légendaires et historiques. Le souci d'exactitude historique concerne la vie de Jeanne d'Arc et la Guerre de Cent Ans. La véracité est cautionnée par l'intertextualité et la mention du Journal du Siège d'Orléans (Boutet de Monvel, 1896: 15): «Ils se sentaient, dit le Journal du Siège, réconfortés et comme désassiègés par la vertu divine de cette simple fille». Du 16 janvier 1412 (naissance de Jeanne d'Arc à Domrémy) au 30 mai 1431 (mort de Jeanne d'Arc), l'album séduit par une iconographie chatoyante qui flatte l'œil par l'élégance du trait. La

\footnotetext{
${ }^{11}$ Le récit se présente sous la forme suivante: pages 5 à 7, les origines de Jeanne d'Arc et l'illumination -pages 8 à 12-, la rencontre avec Charles VII qui lui accorde sa confiance -pages 13 à 32-, la sainte guerrière, ses victoires sur les Anglais, le sacre de Charles VII à Reims le 16 juillet 1429, l'adoration de Jeanne d'Arc -pages 33 à 37-, la défaite due à l'indolente lâcheté de Charles VII -pages 38 à 47-, l'emprisonnement, la torture et la mort de Jeanne d'Arc trahie. La structure du texte fait jaillir le leitmotiv issu de «magnitudo parvi» cher à Hugo.
} 
rigueur des compositions, l'équilibre du texte et de l'image sont un véritable plaisir pour l'œil. Le soin particulier accordé aux costumes par l'artiste reflète le drapé contemporain. La lumière qui les nimbe, le raffinement des motifs et de l'architecture, illuminent l'album à travers un voile de brume qui baigne le drame de l'héroïne «dans une légère pénombre de sérénité mystique» (Embs, 2000: 204). Cette technique qui présente des liens de parenté avec les préraphaëlites anglais s'inscrit dans la mouvance des symbolistes et introduit l'enfant dans un monde familier où il se reconnaît grâce à un esprit concret, simple qui tempère la complexité allégorique.

Les œuvres de Boutet de Monvel s'inscrivent à l'instar de celle de Job, dans le prolongement de l'imagerie populaire: elles offrent à l'enfant une iconographie raffinée qui chante les exploits des grandes figures historiques françaises. L'avènement de la Troisième République, la renaissance de l'économie lors de l'Exposition Universelle de 1878, la réorganisation de l'armée et les réformes de l'Instruction constituent un terreau fertile à l'éclosion des livres d'histoire illustrés: Clio inspire les auteurs qui adaptent le passé aux exigences du présent en métamorphosant le discours historique en une apologie nationale.

\section{Jacques Onfroy de Bréville: Napoléon ou la renaissance française}

C'est dans ce contexte que Jacques Onfroy de Bréville ${ }^{12}$ (plus connu sous le pseudonyme de Job) connaît la consécration. Son interprétation des chevauchées impériales commentées par Georges Montorgueil s'adresse aux plus jeunes par des tableaux pédagogiques. Il décline le passé à l'aide de figures historiques comme Napoléon, mais en oubliant les désinences malheureuses des défaites de Russie, de Waterloo et des Cent Jours. L'inflexion autoritaire du héros conquérant est oubliée au profit de la légende dorée du vaincu. Le souci d'exactitude dans le dessin des costumes confère à ses œuvres une véritable valeur documentaire. Sa jeunesse influe sur la genèse de son œuvre. Agé de douze ans lorsque les Allemands envahissent le France et entrent dans Paris, il conservera une aversion pour les idées révolutionnaires responsables selon lui de l'humiliation du Traité de Francfort.

Sa production s'apparente à une publication parascolaire en adéquation avec les valeurs de la droite ultra. Conscient du pouvoir mnémonique de l'image, il joue de «la réduction épique et vériste du dessin», et attire le regard du lecteur enfantin par «l'inflation pittoresque et la cristallisation anecdotique». Il est persuadé qu'elles facilitent la compréhension et l'appréhension d'un personnage comme Napoléon. Claude-Anne Parmégiani (1989) rappelle sa dérive nationaliste, nourrie de la pensée de Barrès et sa stigmatisation des prisonniers allemands durant la Première Guerre Mondiale ainsi que la dérive raciste à laquelle conduit sa théorie de la morphopsychologie ${ }^{13}$. L'utilisation du format à l'italienne ${ }^{14}$ par Job accentue la perception linéaire du temps dilaté. La voix «off» du narrateur imitée

\footnotetext{
${ }^{12}$ Jacques Onfroy de Bréville (1858-1931), dit Job, pseudonyme composé des initiales.

${ }^{13}$ Job est persuadé que le caractère psychologique est déterminé par le type morphologique des prisonniers de la Grande Guerre.

${ }^{14}$ Le cadre de la double page offre une dimension temporelle élargie et forme une séquence qui prélève des éléments dans le passé. La technique procède par panoramas qui, jusqu'à la fin du $18^{\mathrm{e}}$ siècle, relataient la vie d'une figure célèbre par des images commentées.
} 
du cinéma et le style emphatique de Montorgueil accusent le réalisme de scènes en hypotypose. Rares sont les auteurs illustrateurs aussi conscients de l'herméneutique de l'image.

La consultation du bel album de Boutet de Monvel dédié à Jeanne d'Arc et de quelques spécimens de Job provoque un ravissement esthétique. Mais le trait et le verbe révèlent aussi le regard partial et sélectif des concepteurs dont la mentalité est indéniablement cocardière. Les charges héroïques au souffle épique redorent le blason de l'armée française. La distanciation temporelle prête aux héros de l'Empire un rôle symbolique: ils incarnent la France en marche vers la liberté et se substituent à Marianne. Avec Job l'espace plastique envahit la typographie et opère une fusion entre le dessin et le récit. L'illustrateur applique avec dextérité et finesse les consignes données par Victor Duruy sur la prépondérance de l'image en tant que support pédagogique. Mais surtout il ne peut qu'adhérer au point de vue de Madame Pape-Carpantier qui déclare aux instituteurs réunis à la Sorbonne en 1867: «Quand un enfant ouvre un livre, la première chose qu'il fait ce n'est pas de regarder les pages écrites, mais de regarder s'il y a des images [...], lesquelles, qu'il sache lire ou non, lui disent toujours quelque chose» (Robichon, 1984: 22).

Les ouvrages de Job ont renforcé le sentiment nationaliste. Napoléon devient l'incarnation du redressement national et l'espoir de la revanche. C'est pourquoi Job lui consacre l'essentiel de son travail. Il poursuit l'œuvre du chantre de la pédagogie patriotique des années 1880, Paul Déroulède. Il est vrai que le Premier Empire et l'épopée napoléonienne constituent une riche matrice génétique tant en matière de récits que d'illustrations. Job s'emploie à honorer la mémoire de l'Empereur tout en servant la République. Le Grand Napoléon des petits enfants révèle son art tout en saveur, fait de traits sinueux et fins aux contours imprécis, de délicates couleurs aquarellées. Job parachève sa vision de l'histoire de France avec Jouons à l'histoire: il y imite les techniques narratives des manuels d'histoire en inventant un jeune héros, Jean, écolier studieux, passionné par l'histoire de France qui se déroule sous ses yeux grâce à des tableaux anecdotiques. Après 1890, Napoléon exécré en la personne de «Napoléon le Petit», avatar négatif de son oncle fameux, devient cependant le symbole phare de la Troisième République et Jeanne d'Arc rallie les causes pédagogiques et catholiques en faisant l'unanimité. Une même ferveur unit la Nation autour de la «Sainte Patronne de la Revanche». Boutet de Monvel, Job et Montorgueil alimentent l'imaginaire enfantin collectif par leurs productions. Ils contribuent au consensus national autour d'idéaux communs à la veille de la Première Guerre Mondiale. Ils mettent la connaissance historique à la portée du plus grand nombre et innervent le sentiment d'appartenance à une communauté nationale par le biais de figures mythiques qu'ils ressuscitent.

Ces vulgarisateurs que sont les manuels scolaires ou les albums précédemment étudiés confirment l'instrumentalisation du passé à des fins idéologiques. Ils accentuent l'engagement mémoriel à travers l'évocation de combats politiques, religieux et la «panthéonisation» de figures illustres. Ils dépassent les clivages entre les nostalgiques de l'âge d'or assimilé à l'Ancien Régime et les défenseurs de la liberté, héritiers des révolutionnaires. Ils tirent parti de l'illustration et participent 
à l'acculturation guerrière au même titre que les historiens populaires, les écrivains pédagogues. Leur avantage réside dans la sensibilisation de l'enfant à l'histoire par l'image: avec le crayon et le pinceau, ils excellent dans l'art de s'adresser à l'intelligence, au cœur et à la sensibilité des enfants. Parallèlement à cette littérature enfantine imagée et profondément patriotique, se développe une littérature romanesque juvénile dont les auteurs à l'instar d'Erckmann-Chatrian, de Jacquin, de Paul d'Ivoi ou du capitaine Danrit, comptent parmi les précurseurs d'une littérature belliqueuse et partisane qui alimentera les bibliothèques des écoles et des familles pendant la Grande Guerre.

\section{Bibliographie}

Amalvi, C. (2001): Les héros de l'histoire de France. Paris, Ed. Privat.

Auge, C. (1901): Grammaire du Certificat d'Etudes. Paris, Larousse.

Auge, C. - Petit, M. (vers 1890a): Premier Livre d'Histoire de France, cours élémentaire. Paris, Larousse, s .d.

_ (vers 1890b): Deuxième Livre d'Histoire de France, cours moyen. Paris, Larousse, s. d.

BoCQUILlon, E. (1907): Pour la patrie. Paris, Vuibert et Nony éditeurs.

Bouchor, M. (1913): Chants Populaires pour les Ecoles, poésies. Paris, Hachette.

Boutet de Monvel, M. (1896): Jeanne d'Arc. Paris, Plon-Nourrit et Cie.

COMBARIEU, J. (1914): Le Chant choral, cours supérieur. Paris, Hachette.

DuCOUdray, G. (1884): Cours d'histoire, Notions élémentaires d'histoire générale et d'histoire de France, cours supérieur. Paris, Hachette.

Embs, J. M. - Mellot, P. (2000): Le siècle d'or du livre d'enfants et de jeunesse 1840-1940. Paris, Ed. de l'Amateur.

Gauthier - Deschamps (1905): Cours moyen d'Histoire de France, certificat d'études. Paris, Hachette et Cie.

JuRANVILLE, C. (s. d.): Le deuxième livre des petites filles, cours élémentaire. Paris, Larousse, s. d. Ozouf, M. (1984): L'école de la France, Essai sur la Révolution, l'utopie et l'enseignement. Paris, Gallimard.

- (1992): La république des instituteurs. Paris, Gallimard, Le Seuil.

Onfroy de Breville, J. - Montorgueil, G. (1920): Napoléon. Paris, Boivin, 1921.

PARMegiani, C. A. (1989): Les Petits Français illustrés 1860-1940. Paris, Cercle de la Librairie.

RoBichON, F. (1984): Job ou l'Histoire illustrée. Paris, Herscher.

TOUTEY, E. (1907): Lectures primaires. Paris, Hachette. 